\title{
Spontan Spinal Epidural Hematoma in a Patient Receiving Rivaroxaban After Atrial Fibrilation
}

\section{Atilla Kurcelli1, İlker Çöven²}

\section{${ }^{1}$ Başkent University İstanbul Health Application and Research Center, Clinic of Neurosurgery, İstanbul, Turkey} 2University of Health Sciences, Konya Research and Training Hospital, Clinic of Neurosurgery, Konya, Turkey

\begin{abstract}
We present a case of 70 years whom was operated for cervical spinal stenosis and admitted to hospital 1 month after operation with palpitation. Atrial fibrilation was diagnosed and started rivaroxaban. After six-day medication of rivaroxaban, the patient was admitted to the hospital with sudden onset cervical pain and tetraparesis. Magnetic resonance imagings study revealed spinal compression at the level of C3-C6 associated with
\end{abstract}

spontaneous spinal epidural hematoma (SSEH). Emergent surgery was carried out and the hematoma was evacuated. The patient was discharged one week postoperatively with residual neurological sequel.The pathogenesis of SSEH is unclear, but anticoagulant therapy is a known risk factor. It is a relatively rare disorder.

Keywords: Spontan spinal epidural hematoma, rivoraxaban, atrial fibrilation

Meeting presentation: This manuscript presented at $14^{\text {th }}$ International Update in Cardiology and Cardiovascular Surgery (UCCVS) Congress which was held at the Royal Seginus Convention Center in Antalya, Turkey on April 5-8, 2018.

Kırcelli A. Çöven I. Spontan Spinal Epidural Hematoma in a Patient Receiving Rivaroxaban After Atrial Fibrilation. EJCM 2018; 06 (4): 153-156. DOI: 10.32596/ejcm.00019

\footnotetext{
Address for Correspondence: Atilla Kurcelli, Başkent University İstanbul Health Application and Research Center, Clinic of Neurosurgery, İstanbul, Turkey Phone: +90 21655415 00/2022 e-mail: akircelli@baskent.edu.tr

Received: 22.05.2018 Accepted: 28.05.2018 Published: 30.11.2018 


\section{Introduction}

Spontaneous spinal epidural hematoma (SSEH) is a rare entity and generally requires emergent surgery. ${ }^{(1-4)}$ It can be seen at all ages and is more frequent at the $4^{\text {th }}$ and $5^{\text {th }}$ decades. Male/female ratio is 1.4:1. It is difficult to diagnose SSEH because of atypical symptoms. The presenting complaint is generally neck or back pain but SSEH is usually undiagnosed until neurologic deficit develops. The patients with SSEH may present with sudden onset pain with paresthesia or plegia depending on the level of the spinal cord compression. Predisposing factors are advanced age, vascular anomalies, coagulopathies, anticoagulant use, disc herniation and Paget's disease. ${ }^{(3,5-}$ 7) Rivaroxaban is one of the agents currently being used to prevent embolic events due to atrial fibrillation (AF), which directly inhibits the factor Xa. ${ }^{(8)}$

Recently, the CHA 2DS 2VASc (congestive heart failure, hypertension, age $65-74 />75$, diabetes mellitus, stroke/transient ischemic attack/thromboembolism, vascular disease, female sex) score is recommended by many international guidelines as a simple and practical method of assessing stroke risk in such patients. ${ }^{(9)}$

\section{Case Report}

A 70-year-old man with cervical spinal stenosis was admitted to the hospital for an elective posterior C3-45-6 cervical decompression. The patient had a history of hypertension and non-insulin-dependent diabetes mellitus but no history of coagulopathy and bleeding disorders. The coagulation parameters were within normal levels, with an international normalized ratio (INR) of 0.89 , a partial thromboplastin time of 25.4 seconds, and a platelet count of $450.000 / \mu \mathrm{L}$. Moreover, no antiplatelet and anticoagulant therapies were given preoperatively. After successful decompression surgery, the patient was discharged home at the fifth postoperative day without neurologic deficit. One month after discharge, patient admitted to hospital with palpitation complaint and AF
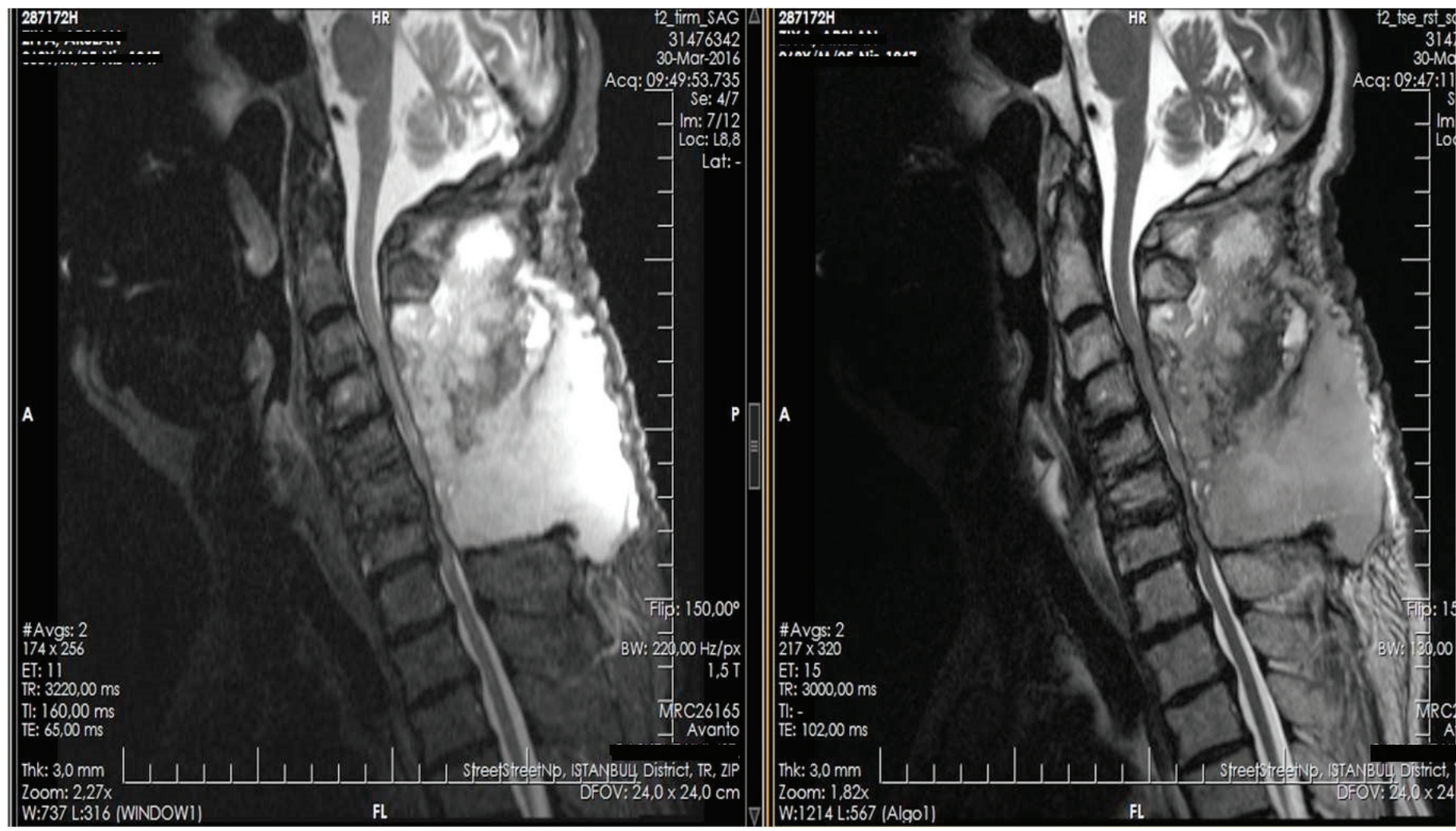

Figure 1. Spinal epidural hematoma after rivaroxabane treatment. T2 weighted sagittal magnetic resonance images 
was diagnosed and started rivaroxaban (Xarelto; Bayer HealthCare AG, Leverkusen, Germany). After six-day medication of rivaroxaban, the patient was admitted to the hospital with sudden onset cervical pain and tetraparesis. Magnetic resonance imaging study revealed spinal compression at the level of C3-C6 associated with SSEH. Emergent surgery was carried out and the hematoma was evacuated during surgery and followed postoperatively three days with drainage tube. Rivaroxaban treatment was stopped. The patient was discharged one week postoperatively with residual neurological sequel.

\section{Discussion}

SSEH is a very rare condition and may be caused directly by trauma and postoperative complications. It is usually more common in the $4^{\text {th }}$ and $5^{\text {th }}$ decades. (10) It is sometimes difficult to diagnose because it is rare, and the symptoms are atypical before converted to catastrophic neurological findings. First symptoms are usually neck and back pain, neurological deficits can be ignored until neurologic deficits occur. Non-traumatic cases are associated with advanced age, low body weight, coagulopathy, anticoagulant therapy, concomitant therapy with aspirin or other nonsteroidal anti-inflammatory drugs, hypertension, vascular malformation, pregnancy, and malignancy. ${ }^{(5,8,10)}$ Most researchers agree that normal activities or maneuvers may cause fluctuations in the intrathoracic and intra-abdominal pressures, leading to an increase in venous pressure, so delicate and valveless epidural venous plexus rupture and bleed. Furthermore, the disruption of a tortuous arterial plexus by traction on nerve roots may cause an arterial source of bleeding by small, disrupted epidural arteries. ${ }^{(3,11)}$ Hemorrhages are usually posterior or posterolaterally associated with hematologic disorders such as hematologic disorders, hemophilia B, and leukemia. The anterior hematomas are usually associated with several risk factors, including anticoagulants, antiaggregants, and vascular malformations. ${ }^{(4,5)}$

We presented a patient with rivoraxaban due to paroxysmal AF. Vitamin $\mathrm{K}$ antagonists such as warfarin in $\mathrm{AF}$ can be used to prevent ischemic stroke. However warfarin is difficult to provide therapeutic INR because of many foods, medicines and pharmacogenomic interactions. Recently, direct anticoagulants such as apixaban, edoxaban, rivaroxaban and dabigatran have become available for anticoagulation therapy. Rivoraxban is factor Xa inhibitor. Deep venous thrombosis prophylaxis with $10 \mathrm{mg} / \mathrm{d}$ of rivaroxaban has a bleeding risk of $0.7 \%$. Currently rivaroxaban has no antidote for the bleeding complications. In J-ROCET AF which evaluated the safety of rivaroxaban compared to warfarin in Japanese people, the rate of critical organ bleeding, intracranial hemorrhages were observed in 5 patients $(0.8 \%)$ in the rivaroxaban group and in 10 patients $(1.6 \%)$ in the warfarin group..$^{(12)}$

\section{Conclusion}

The main treatment to prevent thromboembolic complications of $\mathrm{AF}$ is to use anticoagulant therapy according to CHA2DS2VASc score. Although new agents with supposed lesser side effects are being developed, there may still be complications related to them. Such as, SSEH is a rare but serious condition. Although emergent surgical decompression is the recommended procedure, still some residual neurological sequel may ensue.

Conflict of Interest: No conflict of interest was declared by the authors.

Financial Disclosure: The authors declared that this study received no financial support.

\section{References}

1. Holtås S, Heiling M, Lönntoft M. Spontaneous spinal epidural hematoma: findings at MR imaging and clinical correlation. Radiology 1996;199:40913.

2. Foo D, Rossier AB. Preoperative neurological status in predicting surgical outcome of spinal epidural hematomas. Surg Neurol 1981;15:389-401.

3. Groen RJ, Groenewegen HJ, van Alphen HA, Hoogland PV. Morphology of the human internal vertebral venous plexus: a cadaver study after intravenous Araldite CY 221 injection. Anat Rec 1997;249:285-94.

4. Groen RJ, Ponssen H. The spontaneous spinal epidural hematoma: a study of the etiology. J Neurol Sci 1990;98:121-38. 
5. Horlocker TT, Wedel DJ. Neuraxial block and low-molecular-weight heparin: balancing perioperative analgesia and thromboprophylaxis. Reg Anesth Pain Med 1998;23(6 Suppl 2):164-77.

6. Horlocker TT, Wedel DJ, Benzon H, et al. Regional anesthesia in the anticoagulated patient: defining the risks (the second ASRA Consensus Conference on Neuraxial Anesthesia and Anticoagulation). Reg Anesth Pain Med 2003;28:172-97.

7. Flierl MA, Messina MJ, Mitchell JJ, Hogan C, D’Ambrosia R. Venous thromboembolism prophylaxis after total joint arthroplasty. Orthopedics 2015;38:252-63.

8. Liew A, Eikelboom JW, O'Donnell M, Hart RG. Assessment of anticoagulation intensity and management of bleeding with old and new oral anticoagulants. Can J Cardiol 2013;29:(7 Suppl)34-44.
9. Lowres N, Neubeck L, Salkeld G, et al. Feasibility and cost-effectiveness of stroke prevention through community screening for atrial fibrillation using iPhone ECG in pharmacies. The SEARCH-AF study. Thromb Haemost 2014;111:1167-76.

10. Tawk C, El Haji Moussa M, Zgheib R, Nohra G. Spontaneous epidural hematoma of the spine associated with oral anticoagulants: 3 case studies. Int J Surg Case Rep 2015;13:8-11.

11. Beatty RM, Winston KR. Spontaneous cervical epidural hematoma: a consideration of etiology. J Neurosurg 1984;61:143-8.

12. Hori M, Matsumoto M, Tanahashi N, et al. Rivaroxaban vs. warfarin in Japanese patients with atrial fibrillation - the J-ROCKET AF study. Circ J 2012;76:2104-11. 